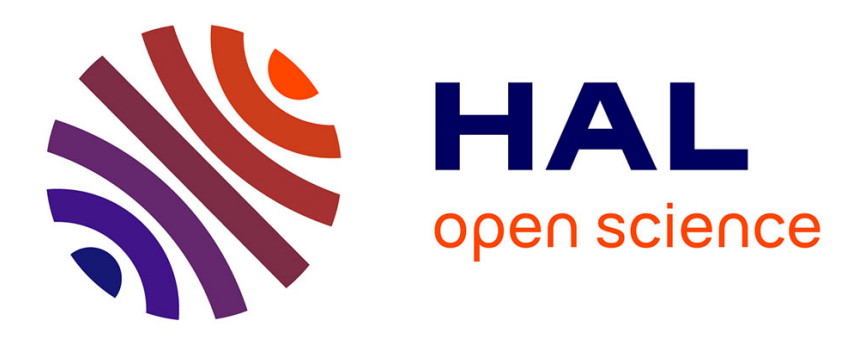

\title{
Représentation du bruit et traitement du signal
}

\author{
O. Macchi, B. Picinbono
}

\section{To cite this version:}

O. Macchi, B. Picinbono. Représentation du bruit et traitement du signal. Revue de Physique Appliquée, 1976, 11 (2), pp.215-225. 10.1051/rphysap:01976001102021500 . jpa-00244051

\section{HAL Id: jpa-00244051 https://hal.science/jpa-00244051}

Submitted on 1 Jan 1976

HAL is a multi-disciplinary open access archive for the deposit and dissemination of scientific research documents, whether they are published or not. The documents may come from teaching and research institutions in France or abroad, or from public or private research centers.
L'archive ouverte pluridisciplinaire $\mathbf{H A L}$, est destinée au dépôt et à la diffusion de documents scientifiques de niveau recherche, publiés ou non, émanant des établissements d'enseignement et de recherche français ou étrangers, des laboratoires publics ou privés. 


\title{
REPRÉSENTATION DU BRUIT ET TRAITEMENT DU SIGNAL
}

\author{
O. MACCHI et B. PICINBONO \\ Laboratoire des Signaux et Systèmes (*), C. N. R. S., \\ Ecole Supérieure d'Electricité, 91190 Gif-sur-Yvette, France
}

\begin{abstract}
Résumé. - Cette conférence passe en revue divers problèmes de traitement du signal en physique et essaye surtout de montrer comment les méthodes dépendent de la connaissance $a$ priori sur le bruit parasite. Ainsi, la représentation correcte du bruit est la condition nécessaire d'un traitement efficace. Ceci est montré sur de nombreux exemples.

Abstract. - In this paper we study some problems of signal processing in Physics. The final objective is to show how the methods of signal processing are depending on the a priori knowledge about the noise. Thus a correct representation of the noise is a necessary condition for an efficient processing. This is pointed out on various examples.
\end{abstract}

1. Introduction. - Le but de cette étude est de présenter certains concepts fondamentaux de traitement du signal pouvant être utiles dans l'analyse et la représentation des nombreux phénomènes physiques qui comportent du bruit. Très sommairement, le bruit peut être défini comme la partie aléatoire de l'observation, qui limite la précision sur la mesure de la grandeur physique que l'on expérimente.

Le bruit est donc de nature très diverse, mais bien souvent par des études préalables, on peut en connaître les moyennes des deux premiers ordres et en particulier pour un bruit stationnaire la répartition spectrale de sa puissance (spectre). Cette simple connaissance permet d'optimiser de nombreux traitements. On étudie en particulier la notion de filtrage adapté pour la détection d'un signal ou la mesure de son amplitude et l'on montre que l'intérêt d'une intégration sur un temps très long est lié à la forme du spectre du signal vers les basses fréquences. On considère ensuite comment cette connaissance spectrale du bruit permet d'optimiser sa représentation à l'aide d'échantillons grâce à la technique de Kahrunen-Loeve.

Dans la deuxième partie de l'étude on envisage diverses situations où la représentation mathématique $\mathrm{du}$ bruit est plus fine qu'une simple description au second ordre. Dans ce cas, les techniques d'extraction du signal hors du bruit peuvent être améliorées en fonction des connaissances que l'on a sur le bruit.

On examine en particulier comment l'hypothèse gaussienne permet de donner un caractère optimal au filtrage adapté introduit précédemment par des méthodes du second ordre. Par ailleuis, on donne quelques exemples de traitement en présence de bruit ponctuel rencontré dans des expériences d'optique.

${ }^{*}$ ) Laboratoire associé à l’Université de Paris-Sud.
Tous illustrent que le traitement du signal doit être adapté à la connaissance que l'on a du bruit.

2. Signal en présence d'un bruit connu au second ordre. - Dans cette première partie, nous étudions un certain nombre de traitements permettant d'extraire le signal utile $S(t)$ du bruit $B(t)$ lorsque la connaissance sur celui-ci se limite à ses deux premiers moments

$$
\begin{aligned}
E(B(t)) & =m(t) \\
E(B(t) B(u)) & =R(t, u) .
\end{aligned}
$$

On pourra souvent supposer que la moyenne $m(t)$ est nulle, sans restreindre la généralité.

2.1 Le FACTEUR TEMPS. - L'idée de départ est qu'une observation sur un intervalle de temps suffisamment long permet d'éliminer le bruit additif par moyenne ou intégration. Cette idée est fondée sur le fait bien connu que si l'on réalise la moyenne de $n$ expériences de type $S+B_{\mathrm{i}}$, la variance du résultat se comporte en $n^{-1 / 2}$, et peut donc devenir aussi petite que l'on veut, si le nombre de répétitions est suffisamment grand.

Essayons de préciser ceci dans le cas d'un signal continu $x(t)$ que l'on veut moyenner. On réalise donc

$$
y(t)=\frac{1}{T} \int_{t-T}^{t} x(\theta) \mathrm{d} \theta
$$

et on voit que le passage de $x(t)$ à $y(t)$ est un filtrage linéaire, une convolution dans un filtre de réponse $R(t)$ valant $1 / T$ dans l'intervalle $[0, T]$ et 0 ailleurs.

Si $x(t)=c$, on a évidemment $y(t)=c$; il est donc intéressant d'étudier $y(t)$ quand $x(t)$ est un bruit stationnaire du second ordre de densité spectrale $\gamma_{x}(v)$

En appliquant la formule classique [1]

$$
\gamma_{y}(v)=|G(v)|^{2} \gamma_{x}(v)
$$


et en calculant $G(v)$ par transformée de Fourier de $R(t)$, on obtient la variance de $y(t)$, qui s'écrit

$$
\sigma_{y}^{2}=\int_{-\infty}^{+\infty}\left(\frac{\sin \pi v T}{\pi v T}\right)^{2} \gamma_{x}(v) \mathrm{d} v .
$$

Il est intéressant d'étudier son comportement lorsque $T \rightarrow \infty \quad$ [2].

Premier cas. - Supposons que $\gamma_{x}(v)$ a une valeur finie non nulle pour $v=0$. On a alors pour $T$ grand

$$
\sigma_{y}^{2} \approx \gamma_{x}(0) \int_{-\infty}^{+\infty}\left(\frac{\sin \pi v T}{\pi v T}\right)^{2} \mathrm{~d} v=\frac{1}{T} \gamma_{x}(0)
$$

qui montre bien une décroissance en $T^{-1 / 2}$ de $\sigma_{y}$ pour $T$ grand.

On peut écrire ceci de manière plus physique en introduisant le temps de corrélation $t_{\mathrm{c}}$ de $x(t)$ défini par exemple par

$$
\gamma_{x}(0)=\int_{-\infty}^{+\infty} \Gamma_{x}(\tau) \mathrm{d} \tau=\sigma_{x}^{2} \int_{-\infty}^{+\infty} \tilde{\Gamma}_{x}(\tau) \mathrm{d} \tau=\sigma_{x}^{2} t_{\mathrm{c}},
$$

d'où l'on déduit

$$
\sigma_{y}^{2} \approx \sigma_{x}^{2} \frac{t_{\mathrm{c}}}{T}
$$

Ceci prouve que c'est le rapport entre $T$ et le temps de corrélation $t_{\mathrm{c}}$ qui fixe la décroissance de $\sigma_{y}$ pour $T$ grand. Ce cas est le plus couramment connu [3], mais il n'est pas le seul.

Second cas. - Supposons qu'au voisinage de l'origine

$$
\gamma_{x}(v)=|v|^{m} f(v)
$$

avec $f(0) \neq 0$. Il faut évidemment supposer que $m>-1$, pour que $E\left(x^{2}\right)<+\infty$, c'est-à-dire que $x(t)$ soit du second ordre. On obtient alors [2] les comportements suivants

$$
\begin{aligned}
-1<m & <1 & \sigma_{y}^{2} & \sim \alpha T^{-(m+1)} \\
m & >1 & \sigma_{y}^{2} & \sim \alpha T^{-2}
\end{aligned}
$$

et on retrouve évidemment (b) comme un cas particulier pour $m=0$.

Ainsi l'avantage du facteur temps dépend essentiellement du comportement de la densité spectrale de $x$ au voisinage de l'origine. On voit en particulier que si $m \rightarrow-1$ (cas du bruit flicker, bien connu dans les fluctuations des oscillateurs), l'intérêt de l'intégration disparaît peu à peu.

Les mêmes résultats se retrouvent en ce qui concerne les mesures de puissance ou de fonction de corrélation.

En fait, l'intégration sur un temps très grand est toujours limitée par les bruits en $1 / v$, cause d'instabilité des phénomènes physiques non éliminables par intégration.

2.2 LE FACTEUR FORME. - On suppose maintenant que le signal a une forme connue et qu'il est perturbé par un bruit additif, centré, connu au second ordre.

Ceci peut se produire dans les situations suivantes, par exemple : bruit,

- extraction d'une lettre d'un alphabet dans un

- extraction d'une raie spectrale de forme connue,

- extraction d'un événement nucléaire,

$-\ldots$

Sans connaissance approfondie sur le bruit parasite, l'outil de base est le filtre adapté qui distord le signal de manière optimale compte tenu des caractéristiques du bruit afin de maximiser le contraste entre le signal et le bruit.

Pour calculer ce filtre, on cherche à rendre maximum le rapport $(S / B)$ à la sortie, rapport défini par

$$
\left(\frac{S}{B}\right)_{\mathrm{s}}^{2}=\frac{S_{0}^{2}\left(t_{0}\right)}{E\left[B_{0}^{2}\right]}
$$

où $S_{0}(t)$ et $B_{0}(t)$ sont les fonctions signal et bruit à la sortie et $t_{0}$ un instant arbitraire. En appliquant les formules classiques de l'analyse harmonique, on trouve, pour un bruit stationnaire,

$$
\left(\frac{S}{B}\right)_{\mathrm{s}}^{2}=\frac{\left[\int G(v) s(v) \mathrm{e}^{2 \pi i v t_{0}} \mathrm{~d} v\right]^{2}}{\int|G(v)|^{2} \gamma_{B}(v) \mathrm{d} v}
$$

où $s(v)$ est la transformée de Fourier du signal d'entrée $s(t), \gamma_{B}(v)$ la densité spectrale du bruit parasite et $G(v)$ le gain du filtre. En appliquant l'inégalité de Schwarz on trouve que $(S / B)_{\mathrm{S}}$ est toujours inférieur à

$$
\int \frac{|s(v)|^{2}}{\gamma_{B}(v)} \mathrm{d} v
$$

valeur atteinte pour

$$
G(v)=k \frac{s^{*}(v) \mathrm{e}^{-2 \pi i v t_{0}}}{\gamma_{B}(v)}
$$

qui est le gain du filtre adapté.

Dans le cas d'un bruit blanc, on obtient

$$
G(v)=k^{\prime}\left[s(v) \mathrm{e}^{2 \pi i v t_{0}}\right]^{*},
$$

d'où par transformation de Fourier

$$
R(t)=k^{\prime} s\left(t_{0}-t\right) .
$$

La sortie du filtre adapté à l'instant $t_{0}$ s'écrit alors

$$
y\left(t_{0}\right)=k^{\prime} \int x(\theta) s(\theta) \mathrm{d} \theta
$$

On voit que c'est en particulier ce qui réalise une détection synchrone dans le cas d'un signal sinusoïdal.

Le filtre adapté peut s'utiliser également lorsque l'on veut mesurer l'amplitude d'un signal de forme connue perturbé par du bruit additif.

Supposons que le signal physique puisse s'écrire :

$$
s(t)=a f(t),
$$


où $f(t)$ est une forme connue et $a$ la quantité physique à mesurer. C'est par exemple le cas d'une impulsion à la sortie d'un photomultiplicateur, $a$ représentant l'amplitude de l'impulsion et $f(t)$ le facteur d'élargissement du P. M.

Le signal $s(t)$ étant superposé à un bruit $B(t)$ centré et connu au second ordre, on désire faire un traitement linéaire permettant d'extraire au mieux $a$. L'entrée du filtre étant $s(t)+B(t)$, la sortie s'écrira $s^{\prime}(t)+B^{\prime}(t)$ ou $a f^{\prime}(t)+B^{\prime}(t)$ et si l'on prend comme critère d'optimalité de rendre $a f^{\prime}(t) / \sigma_{\mathrm{b}^{\prime}}$ maximum, on retrouve l'éq. (1), et par conséquent le filtre adapté.

$\mathrm{Si}$ maintenant les informations de type (6) sont répétitives, compte tenu des propriétés spectrales à l'origine de $B(t)$, on pourra combiner le facteur temps et le facteur forme pour extraire au mieux l'amplitude du signal.

2.3 REPRÉSENTATION DE KaHRUNEN-LoEVE ET SIGNAUX NUMÉRIQUES. - 2.3.1 Représentation d'un signal à l'aide d'échantillons. — Le problème du choix de la représentation à adopter pour décrire au mieux l'information contenue dans un signal aléatoire $x(t)$, sans pour autant accumuler trop de données, apparaît dans de nombreuses expériences physiques. Ainsi lorsque l'observation $x(t)$ doit être traitée sur ordinateur, se pose immédiatement la question du pas d'échantillonnage. On peut ainsi se demander s'il n'existe pas une représentation mieux adaptée à la statistique du signal qu'un échantillonnage temporel. Soit $x(t)$ une fonction aléatoire stationnaire centrée, de covariance.

$$
R(t, u)=\Gamma(t-u) .
$$

Son spectre (ou densité spectrale de puissance) est la fonction $\gamma(v)$ transformée de Fourier de la fonction de corrélation $\Gamma(\tau)$. Il est bien connu que si $x(t)$ est à bande limitée $[-B,+B]$, c'est-à-dire si

$$
\gamma(v)=0 \text { pour }|v|>B
$$

alors $x(t)$ peut être représentée sans erreur à partir des échantillons de $x(t)$ distants de $1 / 2 B$. Nous allons supposer ici que la connaissance de $\gamma(v)$ est d'un type plus fin que (8). Nous supposerons que la fonction de covariance $R(t, u)$ - et par conséquent le spectre $\gamma(v)$ - sont parfaitement connus. Nous verrons qu'alors il existe une repıésentation meilleure que l'échantillonnage temporel, qui ramasse plus d'information à nombre égal d'échantillons.

Considérons maintenant le problème des données aléatoires échantillonnées, mais en surnombre. Soit $\mathbf{x}^{T}=\left(x_{1}, \ldots, x_{n}\right)$ le vecteur ligne fini regroupant ces données. Comment représenter $\mathbf{x}$ de manière à ce que l'information significative soit contenue dans un petit nombre de composantes seulement ? Là encore, cela dépend de l'information que l'on possède $a$ priori sur la statistique du vecteur $\mathbf{x}$. Nous allons supposer, comme dans le cas du signal analogique $x(t)$, que l'on connaît (parfaitement) les moments du second ordre, c'est-à-dire la matrice de covariance

$$
R=E\left(\mathbf{x} \mathbf{x}^{T}\right) \text {. }
$$

Dans la suite du paragraphe 2.3, tous les moments du premier ordre sont supposés nuls.

2.3.2 Représentation de Kahrunen-Loeve (K. L.). La représentation de $K$. L. peut être énoncée de la même manière dans le cas d'un signal analogique $x(t)$, observé sur un temps $[0, T]$ fini, et dans le cas d'un signal vectoriel regroupant un nombre fini $n$ de composantes. Dans le premier cas, pourvu que $R(t, u)$ soit une fonction continue sur $[0, T]^{2}, x(t)$ peut être considéré comme un vecteur aléatoire $|x\rangle$ dans l'espace euclidien $E=\mathbb{R}^{\mathrm{N}}$ - à une infinité dénombrable de dimensions - des fonctions de carré sommable sur $[0, T]$. La seule différence entre les deux cas est donc le nombre de composantes dénombrables, ou fini, qui intervient pour une représentation exacte du vecteur. Nous adopterons la notation de Dirac $|x\rangle$ pour regrouper les cas fini et infini et la représentation de $K$. L. sera dite finie ou infinie suivant le cas.

Dans les deux cas, la covariance est représentée par un opérateur linéaire $R$ absolument continu agissant sur les vecteurs de l'espace $E\left(\mathbb{R}^{\mathbb{N}}\right.$ ou $\left.\mathbb{R}^{n}\right)$.

Définition. - Un système de vecteurs $\left\{\left|\varphi_{i}\right\rangle\right\}$, $i=1,2, \ldots, n$ ou $\infty$ est dit représentation de K. L. du vecteur aléatoire du second ordre $|x\rangle$, si les vecteurs $\left|\varphi_{i}\right\rangle$ forment une base orthonormée de $E$, et si chacun d'eux est vecteur propre de la covariance $R$ de $|x\rangle$ :

$$
\begin{gathered}
<\varphi_{i} \mid \varphi_{j}>=\delta_{i j} \quad \text { (symbole de Kronecker) } \\
R\left|\varphi_{i}>=\lambda_{i}\right| \varphi_{i}>
\end{gathered}
$$

Dans le cas d'un signal analogique $x(t)$ sur l'intervalle $[0, T]$, cette équation est une équation intégrale

$$
\int_{0}^{T} R(t, u) \varphi_{i}(u) \mathrm{d} u=\lambda_{i} \varphi_{i}(t) \quad \forall t \in[0, T] .
$$

La question de l'existence de tels systèmes est résolue par le théorème suivant :

Théorème 1. - Tout vecteur aléatoire du second ordre $|x\rangle$ dont l'opérateur covariance $R$ est continu peut se représenter à l'aide d'au moins une base de K. L.

$$
\left|x>=\sum_{i} \alpha_{i}\right| \varphi_{i}>
$$

Les composantes aléatoires $\alpha_{i}$ satisfont

$$
E\left(\alpha_{i} \alpha_{j}\right)=\lambda_{i} \delta_{i j}
$$

La propriété (14) de non-corrélation entre les composantes est une conséquence immédiate de (10, 11 et 13). Dans le cas d'un signal vectoriel, l'existence d'une base orthonormée formée de vecteurs propres de $R$ est bien 
connue. Dans le cas analogique l'éq. (13) signifie qu'à tout instant $t$ le développement de droite converge en moyenne quadratique vers $x(t)$ et ceci uniformément par rapport à $t$. La preuve se trouve en particulier dans [4]. Parceque les $\left|\varphi_{i}\right\rangle$ forment une base de l'espace $E$, il n'est pas difficile de prouver le théorème qui suit :

Théorème 2. - Les seules bases orthonormées $\left\{\left|\varphi_{i}\right\rangle\right\}$ de l'espace $E$ qui fournissent une représentation du vecteur aléatoire $|x\rangle$ à l'aide de composantes $\alpha_{i}$ non corrélées, sont les représentations de K. L. de $|x\rangle$.

Dans cette représentation, les diverses composantes sont donc telles que leurs informations respectives ne soient pas mélangées. Dans le paragraphe suivant, nous voyons comment l'information est effectivement comprimée.

2.3.3 Optimalité de la représentation de $\mathrm{K}$. L.' - Dans une représentation de $|x\rangle$ du type (13) sur une base $\left\{\left|\varphi_{i}\right\rangle\right\}$ de $E$, les vecteurs $\left|\varphi_{i}\right\rangle$ sont d'autant plus importants que la composante (moyenne) $\alpha_{i}$ est plus grande, c'est-à-dire que $E\left(\alpha_{i}^{2}\right)$ est plus grand.

En appelant $e$ l'énergie (moyenne) de $|x\rangle$

$$
e=E(\langle x \mid x\rangle) \text {, }
$$

il découle du caractère orthonormé des $\left|\varphi_{i}\right\rangle$ que

$$
\sum_{i} E\left(\alpha_{i}^{2}\right)=e
$$

Ainsi le rapport

$$
u_{i}=\frac{E\left(\alpha_{i}^{2}\right)}{e}
$$

mesure le facteur de représentativité du vecteur $\left|\varphi_{i}\right\rangle$ dans la représentation de $|x\rangle$. Les $u_{i}$ sont non négatifs et de somme unité.

Pour comprimer l'information sur le plus petit nombre possible de composantes, il faut que les $u_{i}$ soient concentrées sur quelques composantes, et non dispersées de manière sensiblement égale sur de nombreuses composantes. Or le degré de dispersion de la distribution $\left\{u_{i}\right\}$ est mesuré par l'entropie du système des $\left|\varphi_{i}\right\rangle$ ainsi définie

$$
I\left\{\left|\varphi_{i}\right\rangle\right\}=-\sum_{i} u_{i} \log u_{i} .
$$

$\mathrm{Si}$ au contraire les $u_{i}$ sont très concentrés, l'entropie est très faible (à la limite $I\left\{\left|\varphi_{i}\right\rangle\right\}=0$ lorsque $u_{i}=0$ sauf pour un seul indice $i$ ).

Un système de représentation sera donc optimal au sens de l'entropie, s'il minimise la quantité (18). On montre alors [5] le théorème suivant :

Théorème 3. - Un système orthonormé de représentatation de $|x\rangle$ minimise l'entropie (18) si et seulement si c'est un système de K. L. (l'ordre ne joue pas).
La compression de l'information réalisée par le développement de K. L. se traduit par une autre propriété fondamentale [5]. Pourvu que $x(t)$ ait un comportement raisonnable sur $[0, T]$, un développement du type (13) converge vers $x(t)$. Mais si l'on n'utilise qu'un nombre fini, $m$, de termes (ou un nombre $m<n$ dans le cas d'un signal vectoriel), on fera en général une erreur de troncature. Cette erreur est minimisée par la représentation de K. L. :

Théorème 4. - Un système orthonormé de représentation de $|x\rangle$ minimise l'erreur quadratique moyenne de troncature à $m$ composantes, si et seulement si c'est un système de $\mathrm{K}$. L. ordonné suivant les valeurs décroissantes des valeurs propres $\lambda_{i}$ :

$$
\lambda_{1} \geqslant \lambda_{2} \geqslant \cdots \geqslant \lambda_{i-1} \geqslant \lambda_{i} \geqslant \cdots .
$$

Pour une base orthonormée $\left\{\left|\varphi_{i}\right\rangle\right\}$ donnée, et un nombre $m$ de composantes, cette erreur vaut

$$
\begin{aligned}
\varepsilon\left(\left\{\left|\varphi_{i}\right\rangle\right\}, m\right) & =E\left[\left\langle x-\sum_{i=1}^{m} \alpha_{i} \varphi_{i}\right| x\right. \\
& \left.\left.-\sum_{i=1}^{m} \alpha_{i} \varphi_{i}\right\rangle\right] \\
& =\sum_{i \geqslant m+1} E\left(\alpha_{i}^{2}\right) \\
& =e-\sum_{i=1}^{m} E\left(\alpha_{i}^{2}\right) .
\end{aligned}
$$

C'est donc le développement de K. L. (ordonné suivant (19)) qui maximise le pourcentage d'énergie de $|x\rangle$ pris en compte lorsqu'on tronque le développement à $m$ composantes seulement.

2.3.4 Réalisation pratique. - Pour appliquer ces théories dans la pratique, on peut chercher à résoudre le développement de K. L. infini pour un signal analogique, ou échantillonner le signal et faire un développement de $K$. L. fini.

2.3.4.1 K. L. infini. - Si la covariance $R(t, u)$ est parfaitement connue, on peut songer à résoudre sur ordinateur l'équation intégrale (12). On calcule successivement les valeurs propres $\lambda_{i}$, et l'on s'arrête à l'indice $m$ lorsqu'on constate que l'on a atteint le pourcentage énergétique que l'on désire pour la représentation de $x(t)$. Par exemple, lorsque

$$
\frac{\sum_{i=1}^{m} \lambda_{i}}{e} \geqslant 95 \% \text {. }
$$

On calcule ensuite les $m$ fonctions propres correspondantes $\varphi_{i}(t)$. La résolution est simplifiée dans le cas fréquent où $x(t)$ est stationnaire. Tel est le cas du spectre de Lorentz, très usuel en physique

$$
\gamma(v)=\frac{2 \alpha P}{\alpha^{2}+4 \pi^{2} v^{2}} ; \quad \Gamma(\tau)=P . \mathrm{e}^{-\alpha|\tau|} .
$$


Ce cas est traité en détail dans de nombreux ouvrages. En particulier cf. [6], p. 187. Le cas du spectre rectangulaire est lui aussi bien connu. Les valeurs propres et les fonctions propres dérivent des fonctions d'onde sphéroïdales. Pour ce cas, on peut consulter [7].

D'une manière générale, lorsque le temps d'observation $T$ est très grand devant la largeur de la fonction de corrélation $\Gamma(\tau)$, les fonctions propres sont des fréquences pures $v_{i}=i / T$ multiples de la fréquence $1 / T$, et les valeurs propres sont les valeurs du spectre à ces fréquences $\lambda_{i}=\gamma\left(v_{i}\right)$.

\subsubsection{K. L. fini. - Il n'est pas toujours possible} de résoudre l'équation intégrale. Parfois même on ne connaît pas exactement la fonction $R(t, u)$. On peut seulement estimer la matrice de covariance $R_{N}$ correspondant à $N$ échantillons en progression arithmétique de $x(t)$ sur $[0, T]$. L'estimation se fait à partir d'une suite très longue (bien plus longue que $[0, T]$ ) d'échantillons.

Se pose alors le problème $d u$ pas $\Delta d$ 'échantillonnage, et celui $d u$ nombre $N$ d'échantillons.

$A$ priori, on peut penser qu'on a intérêt à choisir $\Delta$ le plus petit possible. Cependant, outre qu'à partir d'une certaine valeur de $N$, on ne ramasse pratiquement plus d'information supplémentaire, ainsi qu'on va le voir maintenant, la complexité des calculs augmente en $N^{2}$ et des problèmes techniques se posent.

Etude du pas d'échantillonnage optimal pour $T$ donné. - A durée d'observation $T$ donnée, cette étude est faite par Fukunaga et Koontz [8]. Ils considèrent la matrice de covariance $R_{\Delta}(L)$ correspondant à un pas $\Delta$.

$$
L \Delta=T,
$$

et la matrice $R_{\Delta / 2}(2 L)$ correspondant au pas $\Delta / 2$. En appelant $\lambda_{i}^{L}$ et $\lambda_{i}^{2 L}$ les valeurs propres de ces matrices, on a la configuration de la figure 1 , illustrée pour $L=4$.
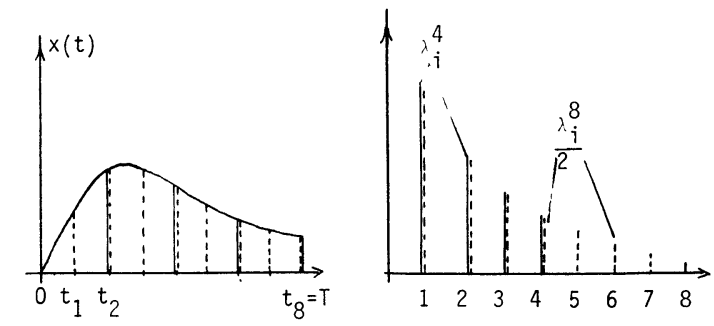

FIG. 1. - Exemple de signal et de valeurs propres.

Il apparaît sur cette figure que la plus grosse partie de l'énergie est concentrée sur les $L$ premières composantes. Pour chiffrer quantitativement cette propriété Fukunaga et Koontz évaluent le rapport

$$
\delta_{2 L}=\frac{\sum_{i=L+1}^{2 L} \lambda_{i}^{2 L}}{\sum_{i=1}^{2 L} \lambda_{i}^{2 L}}
$$

en fonction de la covariance de $x(t)$. Dans le cas stationnaire, ils trouvent

$$
\delta_{2 L} \simeq \frac{\Gamma(0)-\Gamma(T / 2 L)}{2 \Gamma(0)} .
$$

Comme la fonction de corrélation est continue, cette quantité tend vers 0 lorsque $L \rightarrow \infty$. On peut donc toujours s'arranger pour que l'erreur de troncature qui correspond à ne considérer que les $L$ premières composantes soit inférieure à un pourcentage donné. On voit que le pas d'échantillonnage correspondant $\Delta=T / L$, ne dépend pas du tout de la durée $T$, mais seulement de la fonction de corrélation $\Gamma(\tau)$.

Ainsi dans le cas du spectre de Lorentz (22), avec $T=1 / \alpha($ temps de corrélation de $x(t))$, on a

$$
2 L=6, \quad \delta_{2 L} \simeq 0,04
$$

de sorte que l'on gagnera extrêmement peu d'information sur $x(t)$ en cherchant à la représenter avec plus de 3 coordonnées sur l'intervalle $[0,1 / \alpha]$.

Le critère $(25)$ permet de chiffrer cette idée intuitivement évidente : le pas d'échantillonnage

$$
\frac{\Delta}{2}=\frac{T}{2 L}
$$

doit être choisi en fonction du temps de corrélation de $x(t)$, c'est-à-dire de la largeur de sa fonction de corrélation $\Gamma(\tau)$.

Structure de la matrice $R_{\Delta}(L)$, pour $\Delta$ donné. Cette étude est faite par Grenander et Szego [9] pour un processus stationnaire. Soit $\gamma^{\Delta}(v)$ le spectre replié de $x(t)$ défini par

$$
\gamma^{\Delta}(v)=\frac{1}{\Delta} \sum_{k=-\infty}^{+\infty} \gamma\left(v+\frac{k}{\Delta}\right) .
$$

Ce repliement s'effectue dans la bande de fréquences

$$
\left[-\frac{1}{2 \Delta},+\frac{1}{2 \Delta}\right]
$$

correspondant à la fréquence d'échantillonnage (cf. Fig. 2).

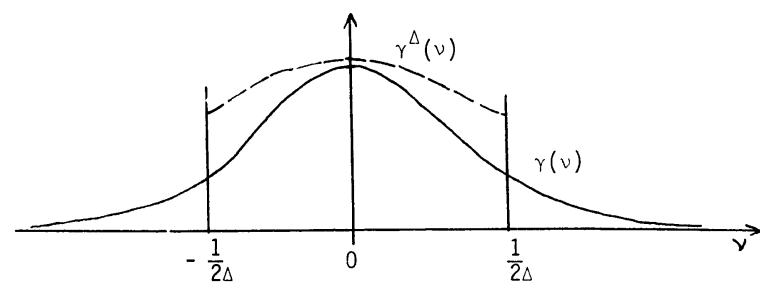

FIG. 2. - Spectre replié

Le pas d'échantillonnage $\Delta$ ayant été choisi à l'aide de l'indice (25), il faut choisir la durée d'observation c'est-à-dire le nombre $L$ d'échantillons, lorsque celui-ci est à notre disposition. Le théorème suivant est un élément de réponse [9] : 
Théorème 5. - Si le spectre replié $\gamma^{4}(v)$ est continu par morceaux, alors les valeurs propres $\lambda$ de $R_{\Delta}(L)$ vérifient

$$
\min _{v} \gamma^{\Delta}(v) \leqslant \lambda \leqslant \max _{v} \gamma^{\Delta}(v) .
$$

Lorsque $L$ tend vers l'infini

$$
\left.\begin{array}{l}
\max \lambda \rightarrow \max _{v} \gamma^{\Delta}(v) \\
\min \lambda \rightarrow \min _{v} \gamma^{\Delta}(v)
\end{array}\right\} .
$$

Naturellement, plus $\Delta$ est petit, plus la dynamique

$$
\left[\max _{v} \gamma^{\Delta}(v)\right]\left[\min _{v} \gamma^{\Delta}(v)\right]^{-1}
$$

est élevée, par conséquent, pour $L$ assez grand, plus

$$
\rho=\frac{\max \lambda}{\min \lambda}
$$

est élevé ; or cette quantité représente la dynamique de la matrice $R_{\Delta}(L)$ et conditionne sa facilité de traitement. Plus $\rho$ est élevé, plus $R_{\Delta}(L)$ sera difficile à traiter, et en particulier difficile à inverser. Or, on verra au paragraphe 3-1, que l'inversion de cette matrice est nécessaire pour la réalisation du filtrage adapté numérique (cf. éq. (31))

$$
\mathbf{H}=\boldsymbol{R}^{-1} \mathbf{S} .
$$

Ainsi, en présence d'un problème de ce type, on doit se garder de la réaction immédiate qui consiste à prendre un pas d'échantillonnage le plus faible possible. Le choix doit être guidé par l'application des considérations précédentes au problème envisagé.

Une étude intéressante de la structure asymptotique de la matrice inverse $R_{\Delta}(L)^{-1}$ pour $\Delta$ fixe, et $L$ tendant vers l'infini est faite par Macchi C. [10]. Il montre que cette matrice a une structure stationnaire (aux coins près) et qu'elle peut être obtenue par simple étirement à partir de $R_{\Delta}\left(L^{\prime}\right)^{-1}$, avec $L^{\prime} \ll L$. D'où une méthode très simple pour l'inversion des matrices de corrélation de grande dimension.

3. Mesure d'un signal en présence d'un bruit statistiquement connu à tous les ordres. - Dans cette seconde partie, nous allons examiner sur quelques exemples particuliers comment la connaissance statistique complète du bruit parasite permet d'introduire des modes de traitement plus élaborés que ceux liés à une simple connaissance du second ordre. Nous considérons le problème de la mesure d'un paramètre physique $a$ - éventuellement vectoriel - lorsque l'information est donnée par le résultat aléatoire $\mathbf{x}$ d'une observation (expérience). La connaissance statistique complète du bruit parasite se traduit par la donnée de la loi de probabilité de $x$ conditionnelle à une valeur quelconque du paramètre à mesurer $a, p(\mathbf{x} / a)$, dite fonction de vraisemblance de $a$. Dans ce contexte la meilleure mesure (estimation) du paramètre $a$ basée sur la connaissance de $\mathbf{x}$ est la valeur de $a$ la plus probable, c'est-à-dire

$$
\hat{a}=a / p(\mathbf{x} / a) \text { maximum . }
$$

Dans ce cadre, nous présentons quelques exemples physiques particuliers d'extraction du paramètre $a$.

3.1 Bruit gaussien. - Le bruit gaussien fera une très bonne transition entre la première et la seconde partie. Il est en effet bien connu que si l'on fait l'hypothèse gaussienne, les propriétés du second ordre déterminent entièrement la loi de probabilité [1].

En particulier, si le bruit est échantillonné, de moyenne $\mathrm{m}$ et de matrice de corrélation $R$, la loi de probabilité du vecteur $\mathbf{x}$, de composantes $x_{1}, \ldots, x_{n}$ s'écrit

$$
p(\mathbf{x})=\alpha \exp -\frac{1}{2}(\mathbf{x}-\mathbf{m})^{T} R^{-1}(\mathbf{x}-\mathbf{m})
$$

où $\alpha$ est une constante ne jouant aucun rôle dans la suite et valant $(2 \pi)^{-n / 2}|\operatorname{det} R|^{-1 / 2}$.

Supposons maintenant que le bruit soit centré $(\mathbf{m}=0)$, et superposé à un signal vectoriel $\mathbf{s}$ de type $a f$ où $f$ est une forme connue (voir éq. (6)).

L'observation $\mathbf{x}=\mathbf{s}+\mathbf{b}$ a pour loi de probabilité

$$
p(\mathbf{x})=\alpha \exp -\frac{1}{2}\left[(\mathbf{x}-a \mathbf{f})^{T} R^{-1}(\mathbf{x}-a \mathbf{f})\right] .
$$

Si l'on veut, comme précédemment, mesurer le paramètre $a$, on peut utiliser la méthode du maximum de vraisemblance consistant à chercher la valeur $\hat{a}$ de $a$ solution de (29). Cette valeur rend (30) maximum. Le calcul est très simple, et l'on obtient

$$
\hat{a}=\frac{\mathbf{x}^{T} R^{-1} \mathbf{f}}{\mathbf{f}^{T} R^{-1} \mathbf{f}} .
$$

Le numérateur qui est le produit scalaire du vecteur observation $\mathbf{x}$ par le vecteur $R^{-1} \mathbf{f}$ s'interprète comme la sortie du filtre adapté échantillonné dont il a été question au paragraphe précédent.

On voit donc que le filtre adapté, qui peut être introduit uniquement par des considérations du second ordre, est de plus une opération optimale en hypothèse gaussienne. Ce résultat n'est pas tellement surprenant, puisque le second ordre caractérise le bruit dans le cas gaussien.

Donnons maintenant un exemple d'application correspondant à ce que nous avons vu au paragraphe 2.1 sur le facteur temps. Supposons que l'observation complète soit de la forme $\mathbf{m}+\mathbf{b}$, où $b$ est un bruit centré et $\mathbf{m}$ le vecteur

$$
\mathbf{m}^{T}=m[1,1, \ldots, 1]=m \mathbf{r}^{T} .
$$

On veut mesurer $m$, qui est la grandeur significative. L'intégration développée au paragraphe 2.1 consiste à réaliser

$$
\hat{m}_{1}=\frac{1}{n} \sum x_{i}=\frac{\mathbf{x}^{T} \mathbf{r}}{\mathbf{r}^{T} \mathbf{r}},
$$

qui est l'analogue numérique de (2.1). 
Par contre la prise en compte du caractère gaussien et la connaissance de $R$ permettent d'introduire l'estimateur optimal

$$
\widehat{m}_{2}=\frac{\mathbf{x}^{T} R^{-1} \mathbf{r}}{\mathbf{r}^{T} R^{-1} \mathbf{r}}
$$

Cet estimateur est supérieur au précédent, et l'on peut chiffrer cette supériorité qui dépend évidemment de la structure de $R$. En particulier si $R$ est diagonale et stationnaire les deux estimateurs sont équivalents.

3.2 BRuits PONCTUELS. - Dans ce paragraphe, nous donnons deux exemples de bruits ponctuels et nous montrons comment on peut faire sortir le signal d'un tel bruit. Ces exemples se présentent en Optique, lorsque les signaux traités sont des flux lumineux faibles et qu'on a atteinte au niveau quantique grâce à un détecteur sensible de photons, par exemple un photomultiplicateur (P. M.) (cf. Fig. 3).

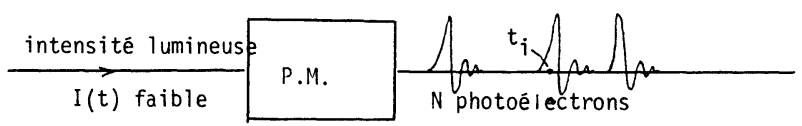

Fig. 3. - Détection de signaux lumineux faibles.

La grandeur observée $\mathbf{x}$ est alors constituée par le nombre aléatoire $N$ de photoélectrons détectés, et éventuellement par leurs positions $t_{1}, \ldots, t_{i}, \ldots, t_{N}$ dans le temps. Le bruit est constitué par le caractère aléatoire de ces photoélectrons alors même que l'intensité lumineuse $I(t)$, grandeur à mesurer, serait déterministe et connue. La fonction de vraisemblance (29) est donc ici la loi de probabilité

$$
p\left(N ; t_{1}, \ldots, t_{N} / I(t)\right) .
$$

3.2.1 Bruit poissonnien (exemple). - On étudie le profil de la lumière diffractée par un cristal autour de l'angle $\alpha_{0}$ où se trouve le maximum du pic de diffraction (cf. Fig. 4).

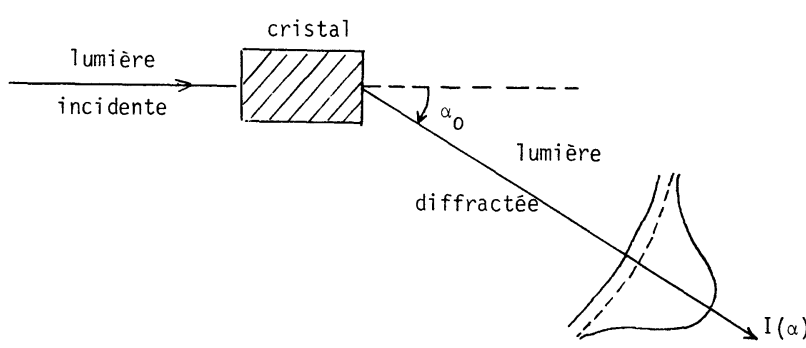

FIG. 4. - Diffraction par un cristal.

La lumière diffractée dans chaque direction $\alpha_{i}$ est très faible et doit être détectée par un P. M. S'y ajoute le fond continu de lumière diffusée d'intensité constante $b$. Ainsi l'intensité lumineuse dans la direction $\alpha_{i}$ est

$$
I\left(\alpha_{i}\right)=b+f\left(\alpha_{i}\right) .
$$

Pour mesurer le profil $f(\alpha)$ et le bruit $b$, on n'a comme observation que l'ensemble des nombres aléatoires $N_{1}, \ldots, N_{k}$ de photons détectés dans chacune des $k$ directions explorées, que l'on regroupera dans un vecteur $\mathbf{N}$ à $k$ dimensions. Les variables $N_{i}, i=1, \ldots, k$, sont poissonniennes, de valeur moyenne (36). Si l'angle entre deux directions consécutives n'est pas trop faible, elles peuvent être considérées comme indépendantes. Dans ces conditions, la fonction de vraisemblance est immédiate :

$$
P(\mathbf{N} / b, f(\alpha))=\exp -\sum_{i=1}^{k}\left(b+f\left(\alpha_{i}\right)\right) \prod_{i=1}^{k} \frac{\left[b+f\left(\alpha_{i}\right)\right]^{N_{i}}}{N_{i} !}
$$

Le choix du profil $f(\alpha)$, et en même temps de la valeur du fond continu $b$, les plus probables, doit être fait en appliquant la méthode de vraisemblance maximum (29) à la probabilité précédente.

Cela est plus facile si le profil de diffraction a été modélisé auparavant et caractérisé par son amplitude $A$ et sa largeur $\sigma$. On essaiera plusieurs formes

$$
\begin{aligned}
& f\left(\alpha_{i}\right)=F_{1}\left(A, \sigma, \alpha_{i}\right)=A \exp -\frac{\left(i-i_{0}\right)^{2}}{2 \sigma^{2}} \quad \text { (Gauss) } \\
& f\left(\alpha_{i}\right)=F_{2}\left(A, \sigma, \alpha_{i}\right)=A\left[1+\frac{\left(i-i_{0}\right)^{2}}{\sigma^{2}}\right]^{-1} \quad \text { (Lorentz) } \\
& f\left(\alpha_{i}\right)=F_{3}\left(A, \sigma, \alpha_{i}\right)=A\left[\frac{\sin \frac{i-i_{0}}{\sigma}}{\frac{i-i_{0}}{\sigma}}\right]^{2} \quad \begin{array}{l}
\text { (fenêtre } \\
\text { optique) }
\end{array}
\end{aligned}
$$

De la sorte, on aura simplement à chercher la valeur du paramètre vectoriel $\mathbf{x}=(b, A, \sigma)$ qui maximise la vraisemblance (37), pour les valeurs $N_{1}, \ldots, N_{k}$ enregistrées. Ces valeurs nous ont été communiquées par P. Becker pour le cristal qu'il étudiait [11] $\left(^{1}\right)$. La maximisation de vraisemblance est facile à réaliser sur ordinateur par une technique algorithmique (méthode du gradient ou méthode de Newton). Dans les 4 exemples traités, la position $\alpha_{0}$ (donc $i_{0}$ ) du maximum du pic était parfaitement connue au préalable. Dans les trois premiers exemples, un deuxième pic était présent dans une direction $i_{1}$ connue, d'amplitude deux fois plus faible que le pic principal. Le profil de diffraction est alors

$$
f\left(\alpha_{i}\right)=F_{l}(A, \sigma, i)+F_{l}\left(\frac{A}{2}, \sigma, i-\left(i_{1}-i_{0}\right)\right),
$$

avec l'indice $l=1,2$ ou 3 correspondant à la forme choisie en (38). Les résultats des estimations sont consignés dans les tableaux suivants

(1) Les résultats de ce paragraphe ont été obtenus en collaboration avec P. Becker et J. M. Pietri. 
EXEMPLE 1

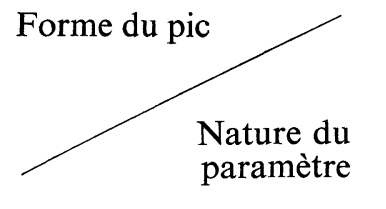

$F_{1}$
$F_{2}$
$F_{3}$

EXEMPLE 2

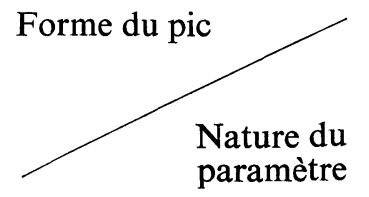

$b$

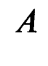

$\sigma$

$\overline{F_{1}}$
$F_{2}$
$F_{3}$

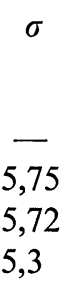

5,3

même qui en résulte. A ce niveau, on voit sur les figures que le profil $F_{1}, F_{2}$ ou $F_{3}$ choisi pour permettre la mise en œuvre de l'estimation n'est pas primordial, pourvu naturellement qu'on ait choisi une forme raisonnable. Ainsi, malgré l'incertitude sur la forme analytique du véritable profil de diffraction, la technique de vraisemblance maximum se révèle extrêmement précieuse pour la mesure de ce profil.

3.2.2 Bruit de Poisson composé (exemple). - Nous présentons maintenant un exemple où l'information n'est plus tirée uniquement du nombre $N$ de photons détectés, mais aussi de leurs positions dans le temps $\left(t_{1}, \ldots, t_{N}\right)$.

La statistique poissonnienne ne rend pas compte de tous les bruits ponctuels rencontrés en optique. En particulier, elle ne rend pas compte de l'effet de groupement présenté par les photoélectrons, et représenté sur la figure 9, dans le domaine temporel.

La fonction $p(\tau)$ représente la densité de probabilité pour que, conditionnellement à la présence d'un photon à l'origine, on trouve un (quelconque) autre photon au voisinage du point $\tau$; $\rho$ est la densité (nombre moyen) de photons par unité de temps. Si la statistique des photons était purement poissonnienne, les nombres de photons au voisinage de l'origine et de l'instant $\tau$ seraient indépendants et $p(\tau)$ serait simplement égal à la densité $\rho$, ce qui n'est pas. L'effet de groupement se traduit $\operatorname{par} p(\tau)>\rho$.

Cet effet provient du phénomène physique suivant : le nombre de photons suit bien une loi de Poisson, mais sa densité est proportionnelle à l'intensité lumineuse $I(t)$ qui est en fait une grandeur fluctuante. Ainsi, les nombres de photons au voisinage de 0 et de $\tau$, qui sont gouvernés par les valeurs $I(0)$ et $I(\tau)$, se trouvent corrélés lorsque le temps $\tau$ est plus faible que la durée de corrélation $\tau_{\mathrm{c}}$ de la lumière (cf. Fig. 9). Le processus est dit de Poisson composé parce que la statistique de Poisson, liée à la détection des photons, se compose avec celle de l'intensité lumineuse $I(t)$. Soit $s$ le coefficient d'efficacité quantique du détecteur. Négligeons son bruit interne pour simplifier (P. M. idéal). Si le processus était poissonnien $(I(t)$ déterministe), il serait facile de voir [12], [13] que la densité de probabilité de l'observation des photoélectrons $t_{1}, \ldots, t_{N}$ sur un intervalle de temps $[0, T]$ serait

$$
p\left(t_{1}, \ldots, t_{N}\right)=s^{N} I\left(t_{1}\right) \ldots I\left(t_{N}\right) \exp -s \int_{0}^{T} I(\theta) \mathrm{d} \theta
$$

Comme cette statistique se compose avec celle de la fonction aléatoire $I(t)$, on a en fait

$p\left(t_{1}, \ldots, t_{N}\right)=s^{N} E\left\{I\left(t_{1}\right) \ldots I\left(t_{N}\right) \exp -s \int_{0}^{T} I(\theta) \mathrm{d} \theta\right\}$.

Le problème que l'on se pose est de retrouver la grandeur aléatoire $I(t)$ à l'aide de l'observation $\left(t_{1}, \ldots, t_{N}\right)$ des photoélectrons détectés. Comme dans les sections 


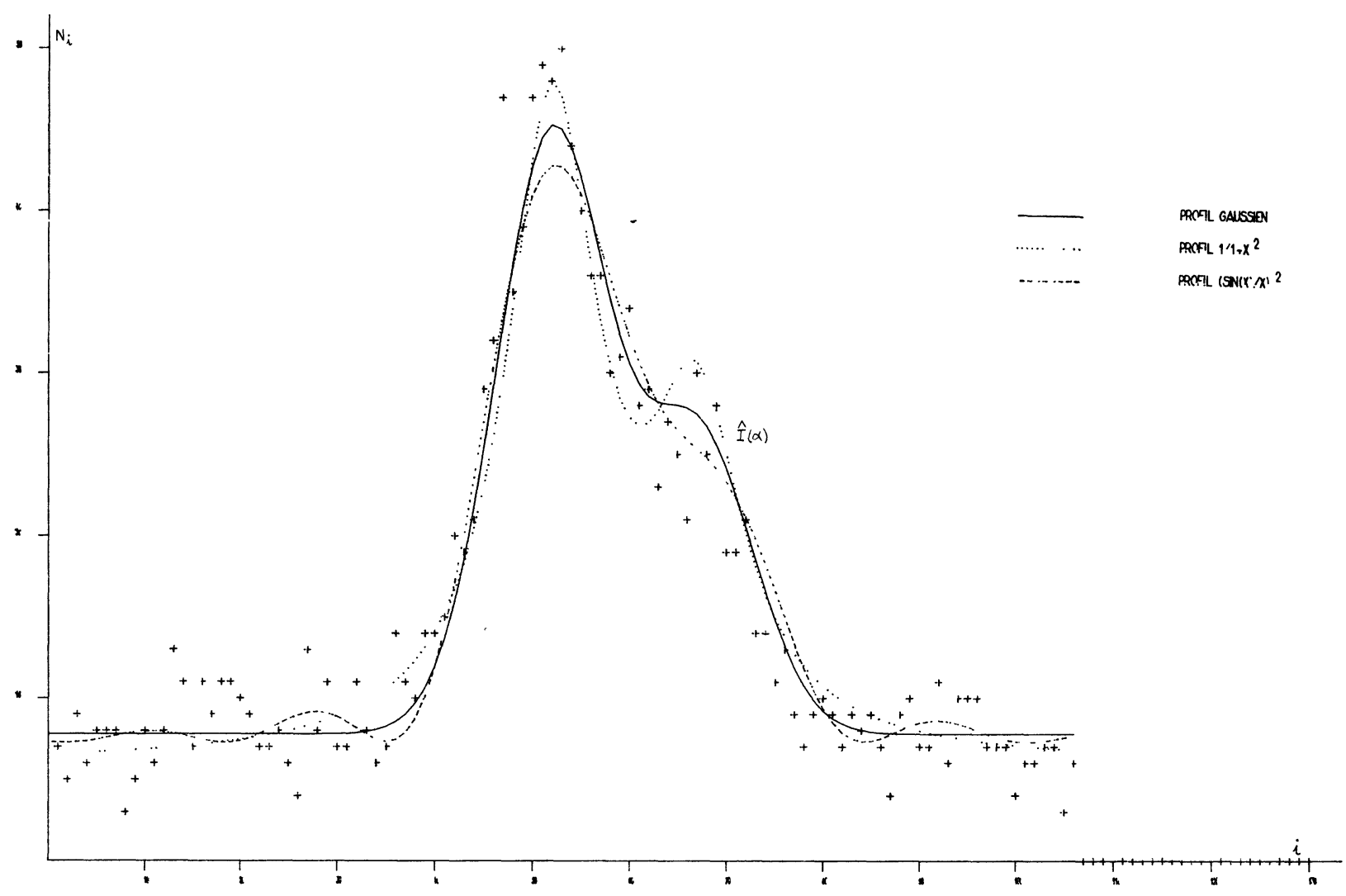

FIG. 5.

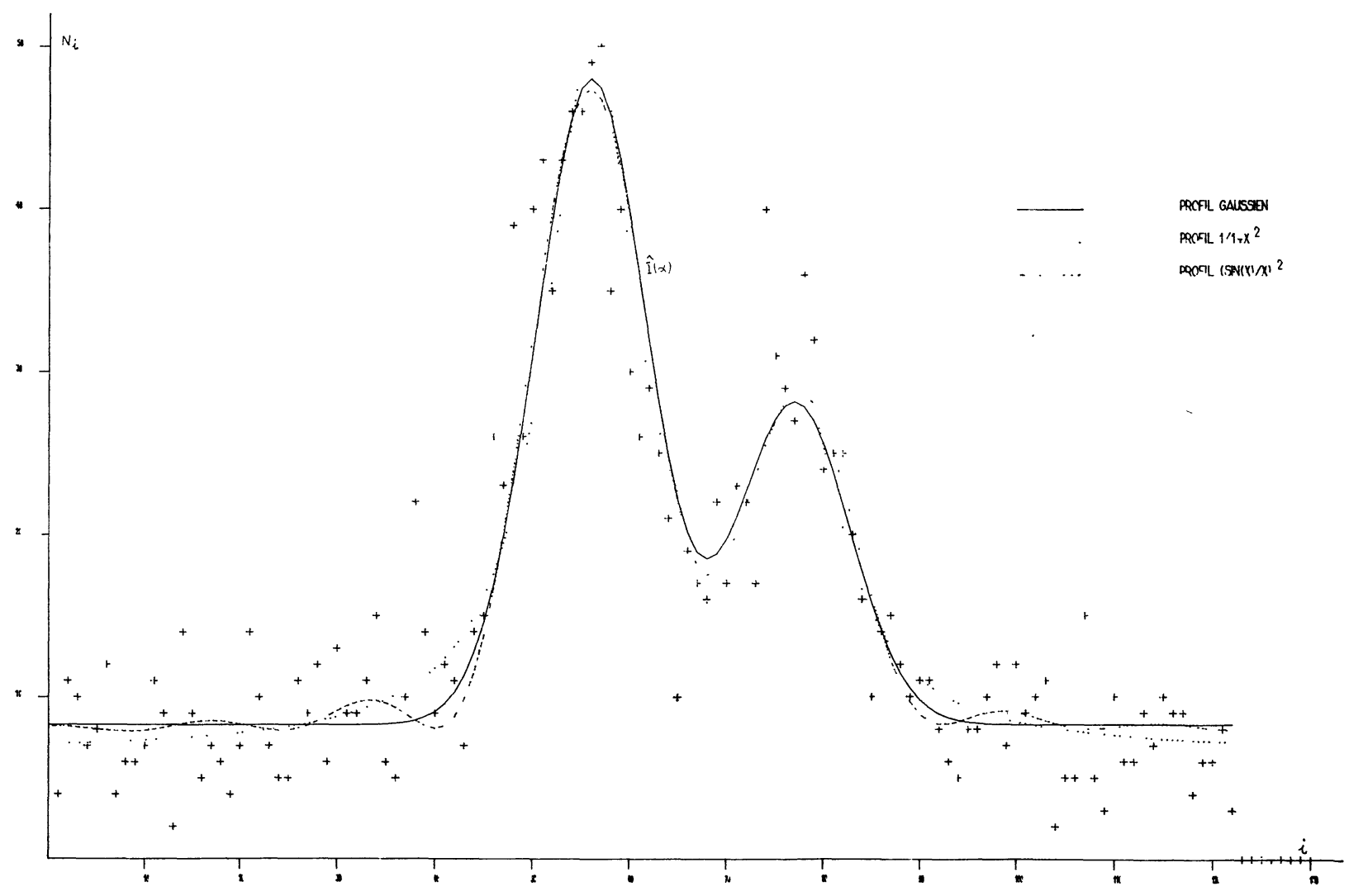

FIG. 6. 
224

REVUE DE PHYSIQUE APPLIQUÉE

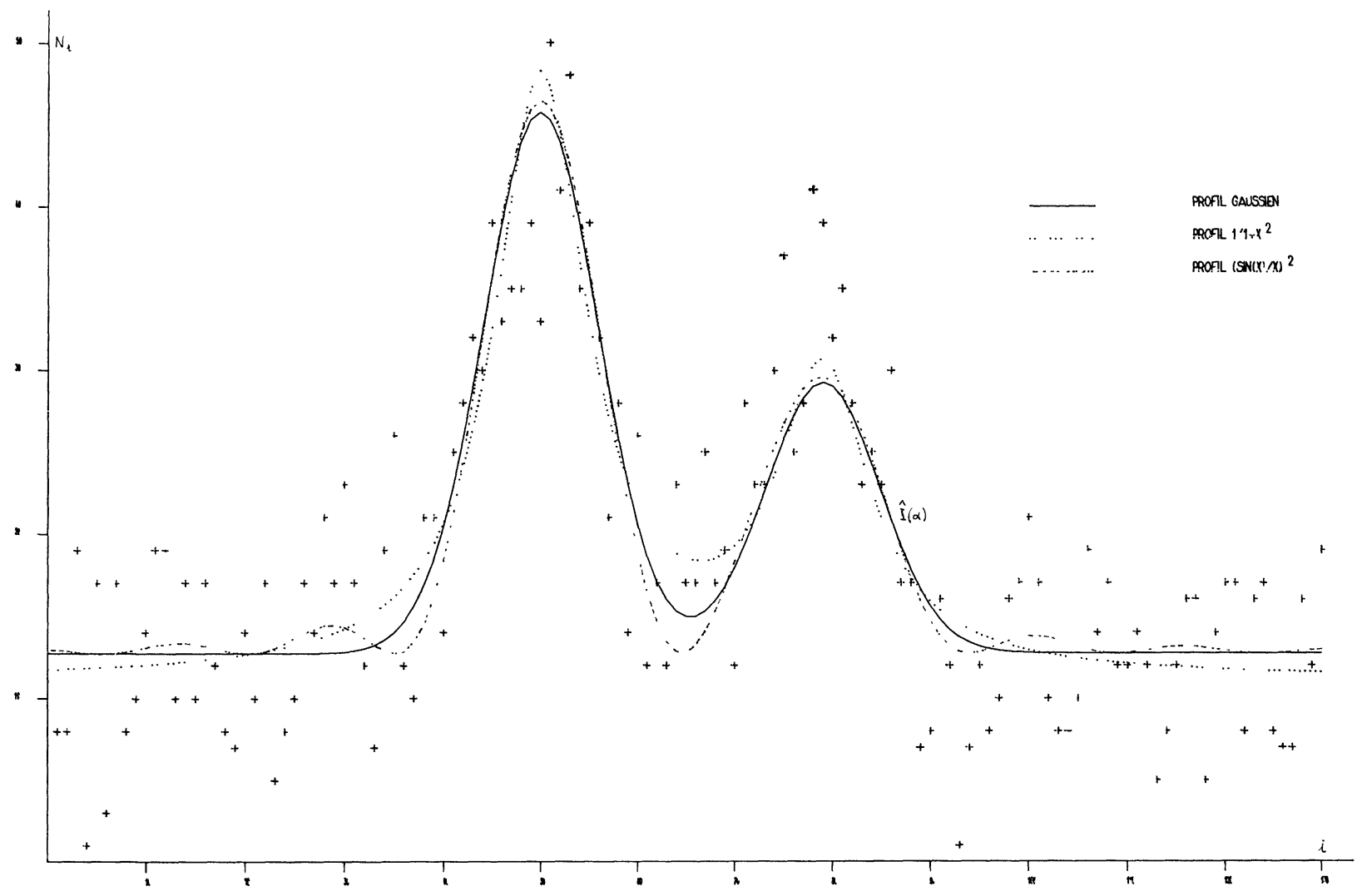

FIG. 7.

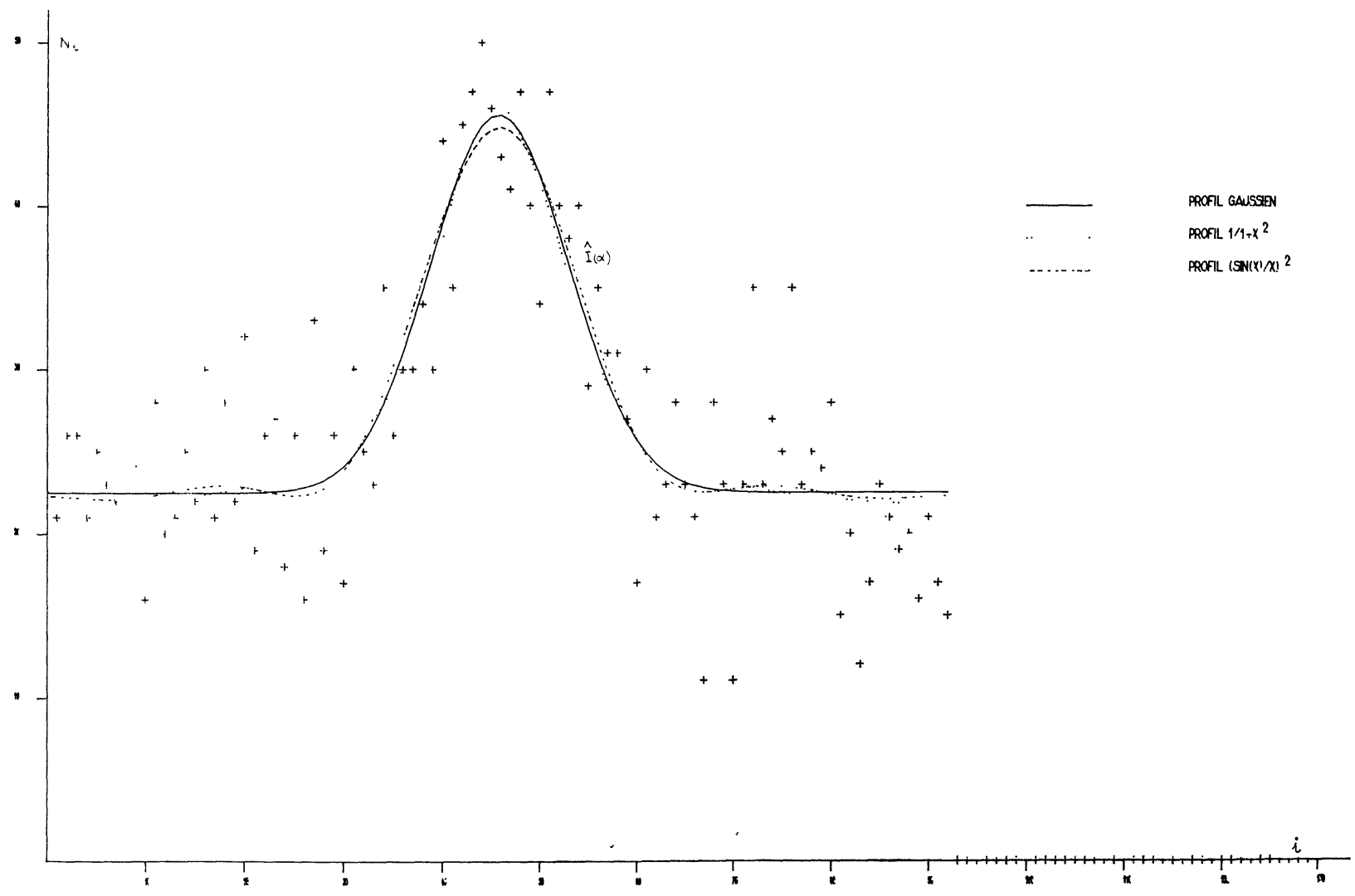

FIG. 8. 


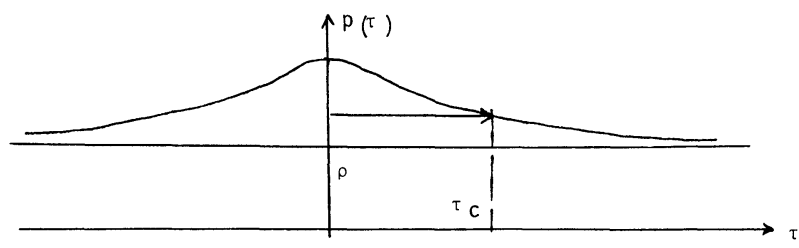

Fig. 9. - Effet de groupement des photons.

précédentes, la meilleure estimation de $I(t)$ doit déduire $I(t)$ de l'observation $\left(t_{1}, \ldots, t_{N}\right)$ en s'appuyant sur la statistique (41) de celle-ci.

Ce problème est traité par $\mathrm{O}$. Macchi et $\mathrm{B}$. Picinbono [12] en utilisant un critère d'erreur quadratique moyenne minimum entre l'estimation $\widehat{I}(t)$ et l'intensité vraie $I(t)$. On trouve

$\widehat{I}(t)=\frac{E\left(I(t) I\left(t_{1}\right) \ldots I\left(t_{N}\right) \exp -s \int_{0}^{T} I(\theta) \mathrm{d} \theta\right)}{E\left(I\left(t_{1}\right) \ldots I\left(t_{N}\right) \exp -s \int_{0}^{T} I(\theta) \mathrm{d} \theta\right)}$

On voit que cette valeur dépend de la loi de probabilité de $I(t)$ qui doit être connue lorsqu'on fait le calcul. Le résultat peut s'exprimer sous forme analytique à l'aide de la covariance complexe $R(t, u)$ du champ électromagnétique lorsque celui-ci est gaussien, comme c'est le cas pour la lumière naturelle ou thermique. Voir [12] Le résultat se simplifie beaucoup si le champ est station- naire et si la densité des photoélectrons détectés est faible, c'est-à-dire, si

$$
s . \rho . \tau_{\mathrm{c}} \ll 1 .
$$

(Dans cette équation, $\rho=E(I(t))$ est l'intensité lumineuse moyenne, et $\tau_{\mathrm{c}}$ est la durée de corrélation du champ, ou ce qui revient au même de l'intensité lumineuse.) On trouve alors

$$
\widehat{I}(t)=\rho+\sum_{i=1}^{N} \frac{\left|\Gamma\left(t-t_{i}\right)\right|^{2}}{\rho},
$$

$\Gamma(\tau)$ étant la fonction de corrélation du champ. Cette formule est illustrée sur la figure 10 .

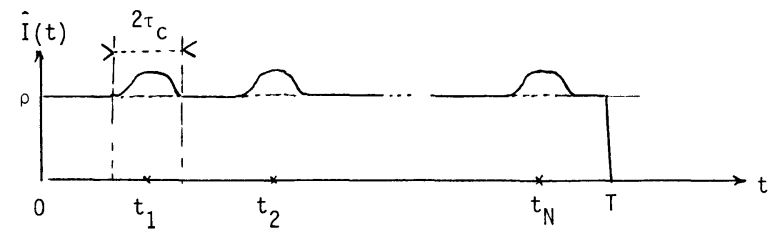

Fig. 10. - Estimation de l'intensité lumineuse à partir des photoélectrons.

La formule (44) et la figure 10 traduisent exactement l'effet de groupement (cf. Fig. 9) : dans un voisinage de largeur $2 \tau_{\mathrm{c}}$ autour de chaque photoélectron détecté, l'intensité lumineuse est estimée plus importante que sa valeur moyenne $\rho$ (et par conséquent la probabilité de détecter un second photon est plus élevée).

\section{Bibliographie}

[1] Picinbono, B., Introduction à l'étude des signaux et Phénomènes aléatoires (Dunod) 1971.

[2] Picinbono, B., Phys. Rev. A 4 (1971) 2398.

[3] Zwanzig, R. et Ailawadi, N., Phys. Rev. 182 (1969) 280.

[4] Courant, R. et Hilbert, D. Methods of Mathematical Physics (Interscience, New York) 1953.

[5] Watanabe, S., «Kahrunen-Loeve expansion and factor analysis. Theoretical remarks and applications". Trans. Fourth Prague Conf. Inf. Theory (1965) 635-660.

[6] Van Trees, H. L., Detection, Estimation and Modulation Theory, Part I (Wiley, New York) 1968.

[7] Slepian, D., Pollak, H. O. and Landau, H. J., "Prolate spheroidal wave functions. Fourier analysis and uncertainty ». Parts I, II, III, Bell Syst. Techn. J. 40 (1961) 43-84, 41 (1962) 1295
[8] Fukunaga, K. and Koontz, W. L. G., « Representation of random processes using the finite Kahrunen-Loeve expansion ». Information and Control 16 (1970) 85-101.

[9] GrenANDER, Szego, Toeplitz forms and their applications (Univ. of Calif. Press) 1958 ch. 5.

[10] MACCHI, C., "Structure de l'inverse d'une matrice de corrélation ». C. R. Hebd. Séan. Acad. Sci. série A (1973).

[11] Blessing, R. H., Coppens, P. and BeCKer, P., J. Appl. Cryst. 7 (1974) 488, "Computer Analysis of Stepscanned X-ray data ».

[12] MACCHI, O. et Picinbono, B., «Estimation and Detection of weak optical signals ». IEEE Trans. on Inf. Theory IT-18 (1972) 562-573.

[13] Bar-David, I., "Communication under the Poisson regime ». IEEE Trans. on Inf. Th. IT-15 (1969) 31-37. 